no reference being made to the Far East or to various lands including or connected with the European centers. Relatively few of the names and achievements of the world's most prominent mathematicians are mentioned, although many more would naturally be expected in any consideration of the Progrès de la Pensée Mathématique. It is to be hoped that M. Pelseneer will carry out on a large scale a further and more complete study of the hidden spirit which has inspired the greatest scholars. For example, what was the spirit that inspired Menaechmus, Deinostratus, Diocles, Heron of Alexandria, and other Greeks whose names are not mentioned? As a single problem, who will undertake to consider the influences which surrounded Leonardo Fibonacci? If a man like Dr. Solomon Gandz, well known for his valuable contributions to Isis and other publications, and with the necessary equipment in the Semitic, Latin and Greek, and modern European languages could find the time to make this study for Fibonacci alone-and I know of no other similar field which promises such interesting results - the work would be of great value and interest.

In the later period there are also such leaders as Napier, Desargues, Barrow, Cotes, Jacobi, Plücker, and Bolyai-to name but a few-each offering a problem of historical interest almost equal to that of Fibonacci, but generally with less of what we may call mathematical romance. Will M. Pelseneer have the time to extend his studies so as to include these and others of equal standing?

The book has a good index of names, some of them of less importance than those mentioned above; but for an index of topics the reader must resort to the less satisfactory Table des Matières.

David Eugene Smith

\title{
MONTEL ON UNIVALENT FUNCTIONS
}

Lȩ̧ons sur les Fonctions Univalentes ou Multivalentes. By Paul Montel. Collection de Monographies sur la Théorie des Fonctions, publiée sous la direction de M. Émile Borel. Paris, Gauthiers-Villars, 1933. iv+159 pp.

This book reproduces, with some modifications, the author's course of lectures delivered at the Sorbonne during the winter of 1929. It contains an exposition of a multitude of interesting results of the theory of univalent functions and of their various extensions. This theory, the origin of which is in the general problem of conformal mapping of simply-connected domains, was given a powerful impetus by the "Verzerrungssatz" of Koebe and Bieberbach. Since then it has attracted the attention and efforts of many mathematicians and at present is perhaps one of the most developed branches of the modern theory of functions of a complex variable. The author starts with classical results, but gives a short account also of the most recent ones, of which, however, he often reproduces only statements, omitting proofs altogether. A consistent use of the theory of normal families which was introduced and developed with great success by the author contributes considerably to the general elegance and unity of exposition.

A brief description of contents follows. Chapter 1 (Univalence et multivalence des fonctions analytiques) contains the fundamental notion of the theory of univalent and multivalent functions together with simple facts concerning convex and star-shaped domains. Chapter 2 (Ordre de multivalence des poly- 
nômes) treats of properties of univalent polynomials. Theorems concerning the roots of a pair of apolar polynomials (Grace-Szegö) play an important role here. Chapter 3 (Familles de fonctions univalentes ou multivalentes) introduces the notion of normal and quasinormal families with various applications, including a solution of the general problem of conformal mapping of simply-connected domains. Chapter 4 (Familles particulières de fonctions univalentes) deals with various applications of the "Verzerrungssatz." A simple solution (due to R. de Possel) of the problem of mapping a multiply-connected domain into a plane with parallel slits should be mentioned here separately. Chapter 5 (Domaines d'univalence ou de multivalence de certaines fonctions) is devoted mainly to a discussion of domains of univalency of entire functions or of functions bounded in the unit circle. An interesting analogy is pointed out here between problems of this type and problems concerning exceptional values of functions (Picard theorem). Chapter 6 (Propriétés extrémales des fonctions univalentes) treats of some simple extremal properties of the mapping function and of its approximation by means of Tchebichev polynomials. Chapter 7 (Domaines couverts par les valeurs des fonctions rêgulières) gives an exposition of results connected with Bloch's theorem. The book ends with a note by M. Cartan on the theory of univalent functions of two complex variables.

The usefulness of the book is somewhat impaired by the presence of numerous misprints and lapses. Thus, after being given the usual definitions of a convex domain $D$ (or a domain star-shaped relative to a point $O$ ) the reader is told that as a point $A$ describes the contour of $D$, the tangent at $A$ turns through an angle of $2 \pi$ (or the vector $O A$ turns through $2 \pi$ ), which rules out such familiar domains as a half-plane (or a plane with a slit along a segment of the axis of reals, extending to infinity). The reader is also told (p. 15) that if there exists a number $\phi$ such that $\operatorname{Re}^{i \phi} z f^{\prime} / f>0$, in $|z|<r$, and if $f(z)$ is analytic in $|z|<r$ and $f(0)=0$, then $f(z)$ is univalent in $|z|<r\left(f(z)=z^{2}\right.$ ?). In another place (p. 10) the reader is told that if a function $f(z)$ is analytic in a closed domain $(d)$ where $f^{\prime}(z) \neq 0$, and if there is no interior point in $(d)$ at which $f(z)$ assumes the same value as on the boundary, then $f(z)$ is univalent in $(d)$ $\left(f(z)=z^{2}, \frac{1}{2} \leqq|z| \leqq 1\right.$ ?). Finally, the footnote 2 on page 5 calls for the following observation. The author refers to his note in the Comptes Rendus (vol. 156 (1913), pp. 1820-1822) concerning an important extension of Cauchy's Theorem, to the effect that if $Z=f(z)=X+i Y$ is continuous in a domain $(d)$ and if the partial derivatives $\partial X / \partial x, \partial X / \partial y, \partial Y / \partial x, \partial Y / \partial y$ exist everywhere in $(d)$ and satisfy Cauchy-Riemann relations almost everywhere in $(d)$, then $f(z)$ is analytic in $(d)$. He states that this result was rediscovered later by Looman (Göttinger Nachrichten, 1923, pp. 97-108). The theorem in question was stated in the author's note above even in a more general form, namely, a necessary and sufficient condition that $p d x+q d y(p, q$ continuous, $\partial p / \partial y, \partial q / \partial x$ exist everywhere) be a total differential is that $\partial p / \partial y=\partial q / \partial x$ almost everywhere. However no proof of this more general theorem has ever been published. Moreover, Looman's proof of the Cauchy theorem is incorrect and the only correct proof of the Cauchy theorem under the general assumptions above, existing in the literature, is due to Menchoff (see S. Saks, Théorie de l'Intégrale, Warsaw, 1933, p. 243).

J. D. TAMARKin 